\title{
On the Energetics of Advection\#dominated Accretion Flows
}

\section{Citation}

Quataert, Eliot, and Ramesh Narayan. 1999. "On the Energetics of Advection\#dominated

Accretion Flows." The Astrophysical Journal 516 (1): 399-410. https://doi.org/10.1086/307097.

\section{Permanent link}

http://nrs.harvard.edu/urn-3:HUL.InstRepos:41384918

\section{Terms of Use}

This article was downloaded from Harvard University's DASH repository, and is made available under the terms and conditions applicable to Other Posted Material, as set forth at http:// nrs.harvard.edu/urn-3:HUL.InstRepos:dash.current.terms-of-use\#LAA

\section{Share Your Story}

The Harvard community has made this article openly available.

Please share how this access benefits you. Submit a story.

\section{Accessibility}




\title{
On the Energetics of Advection-Dominated Accretion Flows
}

\author{
Eliot Quataert! and Ramesh Narayan' \\ Harvard-Smithsonian Center for Astrophysics, 60 Garden St., Cambridge, MA 02138
}

\begin{abstract}
Using mean field MHD, we discuss the energetics of optically thin, two temperature, advection-dominated accretion flows (ADAFs). If the magnetic field is tangled and roughly isotropic, flux freezing is insufficient to maintain the field in equipartition with the gas. In this case, we expect a fraction of the energy generated by shear in the flow to be used to build up the magnetic field strength as the gas flows in; the remaining energy heats the particles. We argue that strictly equipartition magnetic fields are incompatible with a priori reasonable levels of particle heating; instead, the plasma $\beta$ in ADAFs ( $\equiv$ gas pressure divided by magnetic/turbulent pressure) is likely to be $\gtrsim 5$; correspondingly, the viscosity parameter $\alpha$ is likely to be $\lesssim 0.2$.
\end{abstract}

Subject headings: accretion, accretion disks - hydromagnetics - turbulence

\section{Introduction}

Recent work (Narayan \& Yi 1994, 1995ab; Abramowicz et al. 1995; Chen et al. 1995; Nakamura et al. 1997; Manmoto, Mineshige, \& Kusunose 1997; see Narayan, Mahadevan, \& Quataert 1998b, or Kato, Fukue, \& Mineshige 1998 for reviews) has revived interest in a class of hot optically thin accretion solutions first discovered by Ichimaru (1977): advection-dominated accretion flows (ADAFs). In ADAFs, the accreting gas is unable to cool efficiently and most of the energy generated by turbulent stresses is advected onto the central object. As a result, the gas heats up to nearly virial temperatures and adopts a two-temperature configuration (Shapiro, Lightman, \& Eardley 1976; Rees et al. 1982), with the ions significantly hotter than the radiating electrons.

In this paper, we derive and discuss the energy equations for particles, magnetic fields, and turbulence in a two-temperature $\operatorname{ADAF}(\S 2)$. There are several mutually exclusive prescriptions in the literature for treating the energetics of ADAFs (compare, e.g., Esin, McClintock, \& Narayan 1997, hereafter EMN and Manmoto et al. 1997). The differences between these prescriptions lead to significant differences in the predicted spectra of accreting black holes (compare, e.g., Narayan et al. 1998a and Manmoto et al. 1997 for Sgr A*), so it is important to resolve these discrepancies. This is undertaken in Appendix A.

\footnotetext{
${ }^{1}$ equataert@cfa.harvard.edu

${ }^{2}$ rnarayan@cfa.harvard.edu
} 
Non-relativistic ADAFs exhibit a curious tension between two conflicting requirements. On the one hand, energy advection causes the flow to be virial, with a profile of density and temperature $\left(T \propto \rho^{2 / 3}\right)$ that makes a non-relativistic gas nearly isentropic. On the other hand, dissipation of turbulent energy should cause the gas entropy to increase inwards. This tension is particularly relevant for optically thin, two temperature, ADAFs which have seen the most success in applications to real systems (see Narayan et al. 1998b for a review); for such flows, $T_{i} \gg T_{e}$ and the gas is quite accurately approximated as non-relativistic.

In an effort to alleviate this difficulty, Narayan \& Yi (1995b; NY95) suggested that a proper treatment of the flow dynamics should explicitly (if phenomenologically) account for the turbulence/magnetic fields in the flow (since these components are not expected to behave like non-relativistic particles). Using the energy equations derived in $\S 2$, we explore this suggestion in some detail. In $\S 3$ we show that the most straightforward implementation of NY95's suggestion (a constant " $\beta$ " for the flow, which is utilized by many workers in the field) is still incompatible with the turbulent heating of particles. In $\S 4$ we suggest an alternative method of including turbulence in the dynamics of ADAFs which explicitly accounts for particle heating; we present analytical and numerical solutions consistent with this scenario. Finally, in $\S 5$ we summarize and discuss the implications of this work.

\section{The Energetics of ADAFs}

The goal of this section is to derive energy equations for particles, magnetic fields, and turbulence in a two-temperature ADAF (see $\S 18$ of Kato et al. (1998) for a related derivation). Later in this paper (and in Appendix A) we use these equations to clarify several issues relevant to spectral and dynamical models of ADAFs.

Strictly speaking, since ADAFs are nearly collisionless, we should use the Boltzmann equation to analyze their dynamics/energetics. We assume, however, that the use of MHD is justified by the presence of the (turbulent) magnetic field. In particular, this assumes that various wave-particle interactions (pitch angle scattering) maintain approximate isotropy in the distribution functions. With this simplification, the equations governing the structure of the accretion flow are

$$
\begin{gathered}
\frac{\partial \rho}{\partial t}+\nabla \cdot(\rho \mathbf{v})=0 \\
\rho \frac{\partial \mathbf{v}}{\partial t}+\rho(\mathbf{v} \cdot \nabla) \mathbf{v}=-\nabla P+\rho \mathbf{g}+\frac{1}{4 \pi}(\nabla \times \mathbf{B}) \times \mathbf{B}+\eta_{v} \nabla^{2} \mathbf{v}, \\
\frac{\partial \mathbf{B}}{\partial t}=\nabla \times(\mathbf{v} \times \mathbf{B})+\eta_{B} \nabla^{2} \mathbf{B}, \\
\rho T \frac{d s}{d t}=H-q^{-}
\end{gathered}
$$

where $\rho, \mathbf{v}, P, \mathbf{g}, \mathbf{B}, s, \eta_{v}$, and $\eta_{B}$ represent the mass density, velocity, gas pressure, gravitational acceleration, magnetic field strength, particle entropy per unit mass, viscous diffusivity, and magnetic diffusivity in the flow (respectively). In equations (11)-(位),$d / d t$ denotes a Lagrangian derivative while $\partial / \partial t$ denotes an Eulerian derivative. Equation (四) is the sum of the electron 
and ion energy equations, $H$ is a volumetric particle heating rate (due to viscous, resistive, or plasma dissipation mechanisms) and $q^{-}$is the volumetric cooling rate. Below we will discuss the individual ion and electron energy equations.

We emphasize that $H$ is the heating rate of the particles. In ADAFs, $H$ is not necessarily equal to $q^{+}$, the rate at which energy is generated by turbulent stresses/shear in the accretion flow, since some of $q^{+}$may be used to build up the magnetic field strength and turbulent kinetic energy as the gas flows in (see §2.3). Similarly, $s$ in equation (四) is not the total entropy of the fluid, but only that of the particles.

We use mean field theory to derive energy equations for the turbulent flow (see, e.g., Parker (1979) or Speziale (1991) for general discussions of mean field theory and turbulent closure schemes; Balbus \& Hawley (1998) and Kato et al. (1998) give nice applications of this formalism to accretion theory). We assume that each quantity characterizing the flow can be expressed as a sum of an ensemble-averaged ("mean") quantity and a fluctuating quantity, e.g., $\mathbf{v}=\mathbf{U}+\mathbf{u}, \mathbf{B}=\mathbf{B}_{\mathbf{0}}+\mathbf{b}, P=P_{0}+p$, where the fluctuating quantities $(\mathbf{u}, \mathbf{b}, p)$ have zero mean (we denote ensemble-averages by \langle\rangle ). We assume that the turbulence is roughly incompressible, but we do not take $\nabla \cdot \mathbf{v}=0$. Rather, we make the minimal modification to the incompressibility assumption which allows us to treat the compressive heating of particles/field/turbulence; we thus take a static density field, $\rho=\rho(\mathbf{r})$ (i.e., we take $\partial_{t} \rho=0$, but not $d_{t} \rho=0$ ). Another simplification of this work is that we take $\mathbf{B}_{\mathbf{0}}=0$, i.e., we assume that there is no mean magnetic field.

ADAFs are a one scale problem; the vertical scale height $\left(H_{v}\right)$ is of order the local radius in the flow and the inflow time of the gas is of order the rotational period $\left(\Omega^{-1}\right)$. Assuming that turbulent eddies have sizes $\sim H_{v}$ and turnover times $\sim \Omega^{-1}$, this makes the use of a mean field theory somewhat suspect. For now, however, it is the best we can do.

By subtracting the mean versions of equations (2) and (3) from the full equations, one derives equations for $u_{i}$ and $b_{i}$, the fluctuating velocity and magnetic field. Multiplying these equations by $u_{j}$ and $b_{j}$, respectively, yields equations for the evolution of the Maxwell and Reynold's stress tensors. The resulting energy equations for the fluctuating magnetic field and turbulent kinetic energy are given by (see also $§ 18$ of Kato et al. 1998)

$$
\begin{aligned}
d_{t}\left\langle b^{2}\right\rangle & =-2\left\langle b^{2}\right\rangle \partial_{k} U_{k}+2\left\langle b_{i} b_{k}\right\rangle \partial_{k} U_{i} \\
& -\partial_{k}\left(\left\langle u_{k} b^{2}\right\rangle-\eta_{B} \partial_{k}\left\langle b^{2}\right\rangle\right) \\
& +2\left\langle b_{i} b_{k} \partial_{k} u_{i}\right\rangle-\left\langle b^{2} \partial_{k} u_{k}\right\rangle-2 \eta_{B}\left\langle\left(\partial_{k} b_{i}\right)^{2}\right\rangle
\end{aligned}
$$

and

$$
\begin{aligned}
d_{t}\left\langle\rho u^{2}\right\rangle & =-\left\langle\rho u^{2}\right\rangle \partial_{k} U_{k}-2\left\langle\rho u_{i} u_{k}\right\rangle \partial_{k} U_{i} \\
& -2 \partial_{k}\left(\frac{1}{2}\left\langle\rho u^{2} u_{k}\right\rangle+\left\langle p_{t o t} u_{k}\right\rangle-\left\langle\frac{b_{i} b_{k} u_{i}}{4 \pi}\right\rangle-\frac{\eta_{v}}{2} \partial_{k}\left\langle u^{2}\right\rangle\right) \\
& -2\left\langle\frac{b_{i} b_{k}}{4 \pi} \partial_{k} u_{i}\right\rangle+2\left\langle p_{t o t} \partial_{k} u_{k}\right\rangle-2 \eta_{v}\left\langle\left(\partial_{k} v_{i}\right)^{2}\right\rangle,
\end{aligned}
$$

where $p_{t o t}=b^{2} / 8 \pi+p$ is the total fluctuating pressure and $d_{t}=\partial_{t}+U_{k} \partial_{k}$ is the Lagrangian derivative with respect to the mean flow. The physical meaning of these terms is as follows. 
The first line on the right hand side of equations (5) and (6) represents the generation of energy via compression and coupling to shear in the mean flow. The second line gives the divergence of the flux of energy due to turbulence and microscopic resistivity/viscosity. The three terms on the third line represent exchange of energy between the velocity and magnetic components of the turbulence, fluctuating $P d V$ work due to correlations (if present) between the fluctuating pressure and the divergence of the turbulent velocity field, and dissipation by microscopic resistivity/viscosity, respectively.

Taking the mean of equation (四) yields the mean energy equation for the particles,

$$
\begin{aligned}
d_{t}\langle\epsilon\rangle & =-\gamma\langle\epsilon\rangle \partial_{k} U_{k}+\langle H\rangle-\left\langle q^{-}\right\rangle \\
& -\partial_{k}\left(\frac{\left\langle p u_{k}\right\rangle}{\gamma-1}\right)-\left\langle p \partial_{k} u_{k}\right\rangle,
\end{aligned}
$$

where $\epsilon=P /(\gamma-1)$ is the thermal energy per unit volume (and hence $\langle\epsilon\rangle=P_{0} /(\gamma-1)$ ) and $\gamma$ is the adiabatic index of the electron + ion gas; if both species are non-relativistic, $\gamma=5 / 3$, if both are relativistic, $\gamma=4 / 3$, etc..'

A number of different " $\gamma$ 's" will be used in this paper: $\gamma$ refers to the adiabatic index of the electron + ion gas, while $\gamma_{e}$ and $\gamma_{i}$ refer to the individual electron and ion adiabatic indices, respectively. The quantity $\gamma_{t}$ refers to the "adiabatic index" of the turbulence, which is a measure of how efficient flux freezing/compression is at increasing the turbulent energy $(\S 4)$. Finally, $\gamma_{g}$ refers to the total adiabatic index of the fluid, containing contributions from the particles and turbulence $(\S 2.2)$.

\subsection{The Total Energy Equation}

An equation describing the total energetics of the magnetic field, turbulence, and particles is obtained by summing equations (5)-(7), which yields

$$
d_{t} E+\partial_{k} F_{k}=G-\left\langle q^{-}\right\rangle
$$

where

$$
E=\left\langle\frac{1}{2} \rho u^{2}\right\rangle+\left\langle\frac{b^{2}}{8 \pi}\right\rangle+\langle\epsilon\rangle
$$

is the total (turbulent plus thermal) energy per unit volume,

$$
\begin{aligned}
F_{k} & =\left\langle\frac{u_{k} b^{2}}{4 \pi}\right\rangle+\frac{1}{2}\left\langle\rho u^{2} u_{k}\right\rangle+\frac{\gamma}{\gamma-1}\left\langle p u_{k}\right\rangle \\
& -\left\langle\frac{b_{i} b_{k} u_{i}}{4 \pi}\right\rangle-\frac{\eta_{v}}{2} \partial_{k}\left\langle u^{2}\right\rangle-\frac{\eta_{B}}{8 \pi} \partial_{k}\left\langle b^{2}\right\rangle
\end{aligned}
$$

\footnotetext{
${ }^{1}$ In equation ( ( $)$ we have written the particle entropy gradient as $\left\langle\rho T d_{t} s\right\rangle=d_{t}\langle\epsilon\rangle-\gamma\langle\epsilon\rangle U_{k} \partial_{k} \ln \rho$. We use this interchangeably with $\left\langle\rho T d_{t} s\right\rangle=\rho d_{t}\left\langle c_{s}^{2} /(\gamma-1)\right\rangle-\left\langle c_{s}^{2}\right\rangle U_{k} \partial_{k} \rho$, where $c_{s}^{2}=P / \rho$ is the sound speed.
} 
is a turbulent flux (the viscous/resistive contribution is negligible),

$$
G=-\partial_{k} U_{k}\left(\left\langle\frac{1}{2} \rho u^{2}\right\rangle+2\left\langle\frac{b^{2}}{8 \pi}\right\rangle+\gamma\langle\epsilon\rangle\right)-T_{i k} \partial_{k} U_{i}
$$

is the term associated with generating energy, and

$$
T_{i k}=\left\langle\rho u_{i} u_{k}\right\rangle-\frac{\left\langle b_{i} b_{k}\right\rangle}{4 \pi}
$$

is the stress tensor.

\subsection{Approximations}

Equations (5)-(12) are fairly rigorous energy equations for the particles/fluctuations. They are, however, too complicated for some of the calculations of interest to us (e.g., calculating the dynamics of an accretion flow). We therefore make a number of approximations to the mean field energy equations which make them more suitable for simple analytical or numerical calculation. These simplified equations maintain (hopefully) a number of the essential features of the mean field equations.

First, we must provide a closure relation for the third order turbulent quantities (i.e., the turbulent flux). We choose, without much justification, to ignore these terms, setting $F_{k}=0$. Second, we assume that the diagonal components of the turbulent stress tensor are equal, in the sense that $\left\langle b_{r}^{2}\right\rangle \approx\left\langle b_{\theta}^{2}\right\rangle \approx\left\langle b_{\phi}^{2}\right\rangle$, with analogous equations for $\left\langle v_{i}^{2}\right\rangle$. We do not, however, ignore the off diagonal terms in the stress tensor since they are responsible for angular momentum transport. The generalization to arbitrary diagonal components for the stress tensor is given in Appendix B.

We also assume that the flow is azimuthally symmetric, has $U_{\theta}=0$, and rotates uniformly on spherical shells (i.e., $U_{\phi}=r \Omega(r) \sin \theta$, where the rotation rate $\Omega$ is independent of $\theta$; see Narayan \& Yi 1995a). Taking $U_{\theta}=0$ requires that the angular scale height of the flow be roughly independent of radius (Narayan \& Yi 1995a; Abramowicz et al. 1997). With these approximations, we can write, in spherical coordinates,

$$
G=-\left(\frac{5}{3}\left\langle\frac{1}{2} \rho u^{2}\right\rangle+\frac{4}{3}\left\langle\frac{b^{2}}{8 \pi}\right\rangle+\gamma\langle\epsilon\rangle\right) \partial_{k} U_{k}-T_{r \phi} \sin \theta r \frac{d \Omega}{d r}-T_{\theta \phi} \Omega \cos \theta .
$$

This equation demonstrates that, under compression, the velocity component of the turbulence behaves like a gas of adiabatic index $5 / 3$ while the magnetic field behaves like a gas of adiabatic index $4 / 3$. This is a consequence of our assumption of isotropic turbulence.

We express the level of turbulence in the flow in terms of the gas pressure via two parameters:

$$
\beta_{b}=\frac{\langle P\rangle}{\left\langle b^{2} / 8 \pi\right\rangle} \quad \text { and } \quad \beta_{v}=\frac{\langle P\rangle}{\left\langle\frac{1}{2} \rho u^{2}\right\rangle}
$$


We generally take $\beta_{b}=\beta_{v} \equiv \beta$ (i.e., the turbulence is "Alfvenic"). Our definition of $\beta$ is that utilized in the plasma physics literature. A number of workers in the accretion literature (e.g., NY95) define a " $\beta$ " via $\beta_{\text {adv }} \equiv P_{\text {gas }} / P_{\text {tot }}$, i.e., the fraction of the total pressure supplied by the gas. This is related to our $\beta$ by $\beta_{\mathrm{adv}}=3 \beta /(3 \beta+1)$ or $\beta_{\mathrm{adv}}=\beta /(\beta+1)$, depending on whether one defines the magnetic pressure to be $b^{2} / 24 \pi$ or $b^{2} / 8 \pi$.

Finally, we set $\theta=\pi / 2$, i.e., midplane values, in the above expressions; this is a crude but standard - form of height integration. With these assumptions it is rather straightforward to show that the total energy equation for the flow (eq. [8]) takes the form (dropping the \langle\rangle for simplicity)

$$
\rho U_{r} \partial_{r}\left(\frac{v_{t}^{2}}{\gamma_{g}-1}\right)=U_{r} v_{t}^{2} \partial_{r} \rho+q^{+}-q^{-},
$$

where $v_{t}$, the effective isothermal sound speed, is related to the real isothermal sound speed of the gas $\left(c_{s}\right)$ and the turbulent sound speed $\left(c_{t}\right)$ by

$$
v_{t}^{2}=c_{s}^{2}+c_{t}^{2}=c_{s}^{2}\left(1+\beta^{-1}\right), \quad c_{s}^{2}=\frac{\langle P\rangle}{\rho}, \quad c_{t}^{2}=\frac{\left\langle b^{2}\right\rangle}{8 \pi \rho}=\frac{c_{s}^{2}}{\beta} .
$$

The quantity $\gamma_{g}$ is an effective adiabatic index for the flow which includes contributions from the particles, the magnetic field, and the turbulence. For isotropic Alfvenic turbulence, we have

$$
\gamma_{g}=\frac{\gamma+3(\gamma-1) \beta^{-1}}{1+2(\gamma-1) \beta^{-1}}
$$

If we take the ion + electron gas to be nonrelativistic (a good approximation), equation (17) simplifies to

$$
\gamma_{g}=\frac{5 \beta+6}{3 \beta+4}
$$

This expression for $\gamma_{g}$ is slightly different from that of Esin (1997) who found, in our notation, $\gamma_{g}=(15 \beta+8) /(9 \beta+6)$. This is because she neglected the ram pressure of the turbulence, i.e., she set $\beta_{v} \rightarrow \infty$.

The particular expressions for $v_{t}$ and $\gamma_{g}$ in equations (16) and (17) are a consequence of our assumption of isotropic Alfvenic turbulence, the validity of which is highly uncertain. Appendix B generalizes these expressions to the case of arbitrary diagonal components for the stress tensor.

The quantity $q^{+}$in equation (15) is the rate of energy generation in the flow due to coupling between the turbulence and the shear. It is given by

$$
q^{+}=-T_{r \phi} r \frac{d \Omega}{d r}
$$

which can be "closed" using a Shakura \& Sunyaev (1973)-like relation,

$$
T_{r \phi}=-\alpha \rho v_{t} H_{v} r \frac{d \Omega}{d r}
$$

Equation (15) demonstrates an important point. In order to write an energy equation with $q^{+}$(the standard viscous heating term) as a source term, one must consider all energy 
components in the flow (hence the appearance of the "effective" sound speed and "effective" adiabatic index in eq. [15], which contain contributions from the turbulence and the particles). It is, strictly speaking, incorrect to write a particle energy equation with $q^{+}$as a source term, since the particle heating rate, $H$, is, in general, somewhat different from $q^{+}$. The relationship between the "particle" and "total" energy equations is discussed further in Appendix A.

\subsection{The Electron, Ion, and Turbulence Energy Equations}

The mean electron and ion energy equations are given by the individual versions of equation (『) which, utilizing the assumptions given above (steady state, azimuthal symmetry, dropping third order turbulent quantities, etc.), simplify to

$$
U_{r} \partial_{r} \epsilon_{i}=\gamma_{i} \epsilon_{i} U_{r} \partial_{r} \ln \rho+H_{i}-q_{i e}-q_{i}^{-}
$$

and

$$
U_{r} \partial_{r} \epsilon_{e}=\gamma_{e} \epsilon_{e} U_{r} \partial_{r} \ln \rho+H_{e}+q_{i e}-q_{e}^{-},
$$

where $H_{e}\left(H_{i}\right)$ is the energy dissipated out of the turbulence which heats the electrons (ions), $\gamma_{e}\left(\gamma_{i}\right)$ is the monatomic ideal gas adiabatic index for the electrons (ions), $q_{e}^{-}\left(q_{i}^{-}\right)$is the electron (ion) cooling rate, and $q_{i e}$ is the ion-electron energy exchange rate. We have dropped the \langle\rangle for conciseness.

As it stands, however, equations (21) and (22) are not closed since there is no prescription for calculating $H_{e}$ and $H_{i}$. Let us define $\delta_{H}$ to be the fraction of the dissipated turbulent energy which heats the electrons, so that $H_{e}=\delta_{H} H$ and $H_{i}=\left(1-\delta_{H}\right) H$. To get a handle on the particle heating rate, $H$, we note that it is equal to the total energy dissipated out of the turbulence (magnetic and velocity), and thus appears in the energy equation for the turbulence. Summing equations (5) and (6), and employing the various simplifying assumptions outlined in $§ 2.2$, we find that the energy equation for the turbulence simplifies to

$$
U_{r} \partial_{r}\left(\frac{b^{2}}{8 \pi}+\frac{1}{2} \rho u^{2}\right)=\left(\frac{4}{3} \frac{b^{2}}{8 \pi}+\frac{5}{3} \frac{\rho u^{2}}{2}\right) U_{r} \partial_{r} \ln \rho+q^{+}-H
$$

which becomes

$$
2 \rho U_{r} \partial_{r} c_{t}^{2}=c_{t}^{2} U_{r} \partial_{r} \rho+q^{+}-H
$$

for isotropic Alfvenic turbulence (for which $b^{2} / 8 \pi=\rho u^{2} / 2, c_{t}^{2}=b^{2} / 8 \pi \rho$, and the turbulent "adiabatic index" is 1.5).

Equations (23) and (24) state that the rate of change of the turbulent energy as the gas accretes inward is that supplied by compression/flux freezing (the $\partial_{r} \rho$ term) and coupling to the shear in the mean flow $\left(q^{+}\right)$minus the energy dissipated into particle heat $(H)$. We can rewrite these relations schematically as

$$
Q_{t}=q^{+}-H
$$


where $Q_{t}$ is the rate of change of the "turbulent entropy." Note that $H$, the particle heating rate, shows up as a cooling term in the turbulent energy equation.

In ADAFs, just as one cannot ignore the advection term in the particle energy equation, one cannot set $H=q^{+}$and $Q_{t}=0$ in the turbulent equation. We do, however, expect that $H \sim q^{+}$(rather than, say, $H \ll q^{+}$or $H \equiv q^{+}$). This is because of the one scale nature of the turbulent energy equation: the timescale on which shear generates energy (the rotational period) is also the timescale on which energy cascades to small scales to heat particles (i.e., the eddy turnover time), which is also $\sim$ the timescale on which equipartition attempts to be established (the inflow time of the gas).2

For the particular case of isotropic turbulence, $Q_{t} \gtrsim 0$ and thus $H \lesssim q^{+}$. This is because, under compression, isotropic Alfvenic turbulence behaves like a $\gamma=1.5$ gas, i.e., $b^{2} \propto \rho^{1.5}$ (cf eq. [24]). By contrast, for a quasi-spherical flow, equipartition with the gas requires $b^{2} \sim \rho c_{s}^{2} \propto \rho^{5 / 3}$ (§3). Compression (flux freezing) therefore does not supply sufficient energy to keep the turbulence in equipartition with the gas. Some of the energy generated by the coupling between the turbulence and the mean flow $\left(q^{+}\right)$goes into increasing the turbulent energy, while the remainder heats the particles (hence $H \lesssim q^{+}$).

For non-isotropic turbulence configurations, one could have $H \gtrsim q^{+}$. This occurs when the turbulence behaves like a gas of adiabatic index $\gtrsim 5 / 3$ (see Appendix B). Flux freezing/compression then acts to increase the level of turbulence above the equipartition value. In this case, the turbulent energy dissipated in the flow $(H)$ must exceed that generated by shear $\left(q^{+}\right)$, in order for the flow to remain gas pressure dominated (Bisnovatyi-Kogan \& Lovelace 1997).

Many models of two-temperature ADAFs in the literature parameterize the turbulence by assuming a constant $\beta$ for the flow (note that in deriving the above equations we have not employed this assumption). In this case, one can substitute $c_{t}^{2}=c_{s}^{2} \beta^{-1}$ into equation (24) and, knowing the dynamics of the flow (which gives $c_{s}^{2}, \rho, U_{r}, q^{+}$, etc., as a function of $r$ ), calculate the particle heating rate that is implicitly assumed by these models (since $H$ is the remaining unknown in equations [23] and [24]). This is carried out in the next section; surprisingly, and problematically, it turns out that many models in the literature require $H \ll q^{+}$, i.e., most of the energy generated by turbulent stresses $\left(q^{+}\right)$is implicitly assumed to be advected radially by the turbulence $\left(Q_{t}\right)$, rather than heating the particles $(H)$.

\section{The Dynamics of ADAFs}

In this section we reanalyze current treatments for phenomenologically including turbulence/magnetic fields in the dynamics of ADAFs. We show that explicitly decomposing the

\footnotetext{
${ }^{2}$ By contrast, in thin accretion disks the inflow time of the gas is much longer than the the rotational period. To an excellent approximation, then, one can set $Q_{t}=0$ and $H=q^{+}$. This is the turbulent analog of being able to drop the entropy gradient in the particle energy equation, setting $q^{-}=H$ (which becomes the more standard $q^{-}=q^{+}$by the above argument).
} 
energetics of the flow into a turbulent/magnetic component and a gas component, as done in the previous section, leads to a significant reinterpretation of these models.

Consider a steady axisymmetric accretion flow. The dynamics of viscous hydrodynamic accretion can (following Narayan, Kato, \& Honma 1997, hereafter NKH) be described by the following height integrated equations representing conservation of mass, radial momentum, angular momentum, and energy,

$$
\begin{gathered}
\frac{d}{d r}\left(\rho r^{2} H_{\theta} U_{r}\right)=0 \\
U_{r} \frac{d U_{r}}{d r}-\Omega^{2} r=-\Omega_{K}^{2} r-\frac{1}{\rho} \frac{d}{d r}\left(\rho v_{t}^{2}\right), \\
U_{r} \frac{d\left(\Omega r^{2}\right)}{d r}=\frac{1}{\rho r^{2} H_{\theta}} \frac{d}{d r}\left(\frac{\alpha \rho v_{t}^{2} r^{4} H_{\theta}}{\Omega_{K}} \frac{d \Omega}{d r}\right), \\
\rho U_{r} T \frac{d s}{d r}=q^{+}-q^{-} .
\end{gathered}
$$

Anticipating the quasi-sphericity of the flows of interest, we have carried out height integration on spherical shells, so that $H_{\theta}$ is the angular scale height of the flow; where necessary, we take $H_{\theta}=v_{t} /\left(r \Omega_{K}\right) \cdot$. $^{3}$

In the spirit of the previous section, we interpret the pressure (sound speed) and entropy in equations (26)-(29) as effective quantities, containing contributions from both the particles and the turbulence. In particular, we take $v_{t}^{2}=c_{s}^{2}+c_{t}^{2}$ as the sound speed (eq. [16]) and interpret equation (29) as equation (15) (with the effective adiabatic index $\gamma_{g}$ ). It is straightforward to employ the averaging/approximation scheme discussed in the previous section to the MHD momentum equation (eq. 22) and derive equations (27) and (28).

\subsection{Self-Similarity}

In the limit of $q^{-} \ll q^{+}$, cooling provides a rather minor perturbation when calculating the dynamics of the flow. One can therefore set $q^{-}=(1-f) q^{+}$in the energy equation (with $f \sim 1$ ) so that $f q^{+}$of the viscous energy is advected by the flow. In this limit, NY94 showed that, if $\gamma_{g}$ and $f$ are independent of radius, and $\Omega_{K}$ is Newtonian, equations (26)-(29) admit a self-similar solution, with the various flow variables being power laws in radius (note that this entails that $\beta$ is a constant, independent of radius). In particular, $H_{\theta}=$ constant, $U_{r} \propto \alpha r^{-1 / 2}$,

\footnotetext{
${ }^{3}$ In spherical coordinates, balancing pressure and the centrifugal force in the $\theta$ direction implies $\rho \propto$ $\exp \left[-0.5 \Omega^{2} r^{2} \cos ^{2} \theta / v_{t}^{2}\right]$ (e.g., Narayan, Barret, \& McClintock 1997), assuming $v_{t}$ and $\Omega$ to be independent of $\theta$ (this, but not $\Omega, v_{t}$ independent of $z$, is a reasonable approximation for ADAFs). Defining $H_{\theta}$ to be the spherical average of the density, i.e., $H_{\theta}=(4 \pi)^{-1} \int \sin \theta d \theta d \phi \rho$ yields $H_{\theta}=(\pi / 2)^{1 / 2} x \operatorname{erf}\left(x^{-1} / \sqrt{2}\right)$, where $x=v_{t} /(\Omega r)$. This is the correct expression for $H_{\theta}$ for ADAFs. It is, however, numerically cumbersome, so for now we stick with the (more standard) expression given in the text.
} 
$v_{t}^{2} \propto r^{-1}, \rho \propto \alpha^{-1} r^{-3 / 2}$, and $\Omega \propto\left(5 / 3-\gamma_{g}\right)^{1 / 2} \Omega_{K}$ (see NY94 or Narayan et al. 1998b for the complete scalings).

Of particular interest for our purposes is the dependence of $\Omega$ on $\gamma_{g}$. Non-relativistic $\left(\gamma_{g}=5 / 3\right)$ advection-dominated accretion flows tend to be non-rotating. The reason is that the above equations describing the structure of the accretion flow in $1 \mathrm{D}$ exhibit the following "singularity." Approximate hydrostatic equilibrium in the radial direction implies a virial flow, for which $v_{t}^{2} \sim r^{2} \Omega_{K}^{2} \propto r^{-1}$. Together with angular momentum conservation, this implies $U_{r} \sim \alpha r^{-1 / 2}$. Mass conservation then implies $\rho \propto \alpha^{-1} r^{-3 / 2}$. Together, then, mass, radial momentum, and angular momentum conservation impose a $T \propto v_{t}^{2} \propto \rho^{2 / 3}$ density/temperature profile on the flow. The remaining flow variable, $\Omega$, adjusts itself to ensure that the energy equation is satisfied. The profile $T \propto \rho^{2 / 3}$ is, however, an adiabat for a non-relativistic flow with $\gamma_{g}=5 / 3$. This means that there can be no entropy generation as the gas flows inwards, which requires that $\Omega$ and $q^{+}$go to zero.

The remainder of this paper is predicated on the assumption that this singularity as $\gamma_{g} \rightarrow 5 / 3$ is, at some level, real, and is not an artifact of the approximate treatment of the dynamics given above. Height integration schemes differing from ours do not show this behavior (e.g., Chen et al. 1997; Manmoto et al. 1997). We believe that these models, based on cylindrical averaging, are not accurate representations of the quasi-spherical flows of interest, but only $2 \mathrm{D}$ or $3 \mathrm{D}$ calculations will fully resolve this issue.

NY95 proposed that non-relativistic ADAFs (such as the much applied $T_{i} \gg T_{e}$ variety) behave differently from a $5 / 3$ gas because of turbulence (in particular, magnetic fields) in the flow. The "formal" justification for this suggestion is given by the derivation of $\S 2$ (cf eqs. 15][18] and the accompanying discussion). In what follows, we employ the most straightforward implementation of NY95's suggestion; we analyze the dynamics of ADAFs assuming nonrelativistic particles and a constant $\beta$ turbulent/magnetic contribution to the pressure and energy density of the flow.

In this case, the effective adiabatic index $\gamma_{g} \neq 5 / 3$, and the flow can be differentially rotating. This is important since we expect real flows to possess non-negligible angular momentum. If, however, we take the self-similar solution for $\gamma_{g} \neq 5 / 3$ and compute the entropy gradient for a nonrelativistic gas $(\gamma=5 / 3)$, we find $T d s /\left.d r\right|_{\text {gas }}=0$. Accounting for turbulence/magnetic fields to make the flow as a whole $\left(\gamma_{g}\right)$ behave differently from a $5 / 3$ gas does not alter the fact that the gas component of the flow still has $\gamma \approx 5 / 3$ (see Appendix A), and therefore has no entropy gradient in the self-similar regime. It therefore does not alleviate the fundamental tension between being virial and the need for particle heating.

Using the energy equations given in $\S 2$ (see the discussion below eq. [24]), it is straightforward to show that $H=q^{-}=(1-f) q^{+}$and $Q_{t}=f q^{+}$. This implies that, although including constant $\beta$ turbulence in the dynamics of a non-relativistic ADAF allows for differential rotation and entropy generation $\left(q^{+} \neq 0\right)$, it does so by requiring that almost none of the energy generated by turbulent stresses heats the particles (since for $f \sim 1, H \ll q^{+}$). Instead, this energy is assumed to be stored as turbulent energy in the flow, i.e., it is advected by the turbulence, rather than the particles. For the self-similar solution, this conclusion is independent of $\beta$. For larger $\beta$, the turbulent energy is smaller; $\gamma_{g}$ is, however, closer to $5 / 3$ and so $\Omega$ and 
$q^{+}$are smaller as well. The solution always adjusts itself so that the energy generated by the shear is precisely what is required to maintain constant $\beta$ in the flow.

The condition $H \ll q^{+}$means that only a small fraction of the turbulent energy cascades to small scales and heats the particles. This seems unreasonable since intuition and dimensional analysis dictate that ADAFs should have $H \sim q^{+}$(although not $H \equiv q^{+}$; see $\S 2.3$ ).

\section{An Alternative Dynamics: Explicit Inclusion of Particle Heating}

One way to resolve the above contradiction is to give up the assumption of constant $\beta$. Instead, let us assume that the magnitude of the turbulence/magnetic fields in the flow adjusts so as to allow the requisite level of particle heating.

To analyze this scenario, we replace the single energy equation representing "particles plus turbulence" (eq. [15]) with the following two energy equations, the first for the particles and the second for the turbulence,

$$
\begin{gathered}
\rho U_{r} \partial_{r}\left(\frac{c_{s}^{2}}{\gamma-1}\right)=U_{r} c_{s}^{2} \partial_{r} \rho+H-q^{-} \\
\rho U_{r} \partial_{r}\left(\frac{c_{t}^{2}}{\gamma_{t}-1}\right)=U_{r} c_{t}^{2} \partial_{r} \rho+q^{+}-H .
\end{gathered}
$$

The quantity $\gamma\left(\gamma_{t}\right)$ is the particle (turbulent) adiabatic index. For $c_{t}^{2}=b^{2} / 8 \pi \rho$ and $\gamma_{t}=1.5$, equation (31) was derived in $\S 2.3$ (it is equivalent to eq. [24]); here we allow for a slightly more general fluid model of the turbulence by considering a general value of $\gamma_{t}$. Equation (30) is the sum of the individual particle energy equations (eqs. 21] and 22]).

To ensure particle heating, we replace the assumption of a constant $\beta$ with the assumption that a constant fraction $\eta$ of the energy generated by turbulent stresses heats the particles, i.e.,

$$
\eta \equiv H / q^{+}=\text {constant }
$$

$\beta(r)$ is now an output of the dynamical equations. Mass, radial momentum, and angular momentum conservation are still given by equations (26)-(28), taking the total pressure to be the sum of the gas and turbulent pressures, $v_{t}^{2}=c_{s}^{2}+c_{t}^{2}$. To be consistent with previous treatments, we define $q^{-}=(1-f) q^{+}$(although $q^{-}=(1-f) H$ might be a more physical paramatrization). With these definitions,

$$
H-q^{-}=(\eta+f-1) q^{+} \quad \text { and } \quad q^{+}-H=(1-\eta) q^{+} .
$$

As discussed in Appendix B, a crucial assumption of the present analysis is that $\gamma_{t} \lesssim 5 / 3$. This is equivalent to the statement that the effect of compression/flux freezing on the turbulent energy is insufficient to maintain the turbulence in equipartition with the gas. In this case, it is $q^{+}$, the coupling between the turbulence and the shear, which is primarily responsible for increasing the turbulent/magnetic energy as the gas accretes inwards. 


\subsection{Self-Similarity}

We search for analytical solutions to equations (26)-(28) and (30)-(31), assuming that $f$, $\eta$, and $\alpha$ are independent of $r$. For simplicity we take $\alpha^{2} \ll 1$ (almost certainly a reasonable approximation), $\beta \gtrsim 1$ (also likely to be a reasonable approximation), and $\gamma=5 / 3$ (nonrelativistic gas, i.e., ion dominated). The assumption on $\beta$ is needed in order to express all quantities in the solution as power laws in radius (in particular, it allows us to approximate $c_{s}^{2}=v_{t}^{2} /\left(1+\beta^{-1}\right)$ as $\left.c_{s}^{2} \approx v_{t}^{2}\left(1-\beta^{-1}\right)\right)$. With these assumptions it is relatively straightforward to obtain the following approximate solution to the above equations. At a fiducial radius $r_{0}$, let $\beta=\beta_{0}$. For $r<r_{0}$, we then have

$$
\begin{gathered}
v_{t}=\sqrt{\frac{2}{5}} r \Omega_{K} \quad, \quad U_{r}=-\frac{2 \alpha}{5}(3 / 2-a) r \Omega_{K}, \\
\Omega=\Omega_{0}\left(\frac{r}{r_{0}}\right)^{a-3 / 2}, \quad \beta=\beta_{0}\left(\frac{r}{r_{0}}\right)^{-2 a}, \\
\Omega_{0}=\Omega_{K}\left(r_{0}\right)\left(\frac{\left(5-3 \gamma_{t}\right)}{5 \beta_{0}(3 / 2-a) \tilde{f}}\right)^{1 / 2}, \\
\tilde{f}=2(\eta+f-1) / 3+(1-\eta)\left(\gamma_{t}-1\right), \\
a=\frac{\left(5-3 \gamma_{t}\right)(1-f-\eta)}{6\left(\gamma_{t}-1\right)(\eta-1)-4 f}
\end{gathered}
$$

and

$$
\eta=\frac{(1-f)\left(5-3 \gamma_{t}-4 a\right)+6 a\left(\gamma_{t}-1\right)}{5-3 \gamma_{t}-a\left(4-6\left(\gamma_{t}-1\right)\right)}
$$

For $a=0, \eta=1-f$, and the above solution reduces to that of NY94. Equations (34)-(39) are therefore the generalization of the NY94 self-similar solution to the case when particle heating is explicitly included in a flow with non-relativistic particles. We note that, even though the flow has $v_{t}^{2} \propto \rho^{2 / 3}$, which was the origin of the difficulty in NY94's self-similar solution, particle heating is accounted for in the present solution; this is because $\beta \neq$ constant, so that neither the gas nor the turbulence is strictly virial (although their sum is).

The key feature of the above solution is that $\beta$ increases as a function of decreasing $r(a \geq$ $0)$. In the self-similar solution of NY94 all of the energy generated by turbulent stresses goes into maintaining the turbulence in equipartition with the gas (regardless of the magnitude of $\beta$ ). Explicit inclusion of particle heating therefore must (and does) yield subthermal magnetic field strengths. Once some of the energy goes into particle heating, there is insufficient energy available to maintain constant $\beta$ (given the constraints imposed by being virial). The increase in $\beta$ is accompanied by a decrease in $\Omega / \Omega_{K}$ (recall that the NY94 solution has constant $\Omega / \Omega_{K}$ ). The total pressure and radial velocity (and hence density), however, have the same (virial) radial dependence as in NY94.

A priori, we expect $H \sim q^{+}$. We therefore take $\eta=1 / 2$ (equal turbulent and particle heating) as a canonical value. For $f=1$, this yields $a=1 / 14$ and $\beta \propto r^{-1 / 7}$ for $\gamma_{t}=1.5$ and $a=1 / 6$ and $\beta \propto r^{-1 / 3}$ for $\gamma_{t}=4 / 3$ (isotropic magnetically dominated turbulence, for which $c_{t}^{2}=b^{2} / 24 \pi \rho$ ). For an ADAF with a radial extent $\sim 10^{4}$ Schwarzschild radii, $\beta$ changes (over 
the whole flow) by a factor $\sim 4(\sim 20)$ for $\gamma_{t}=1.5(4 / 3)$. Explicit inclusion of particle heating can therefore lead to noticeably subthermal magnetic fields. The precise numerical factor by which $\beta$ changes is, however, quite sensitive to the (poorly understood) choice of $\gamma_{t}$ and $\eta$.

There are two unsatisfying features of this analytical solution. The first is the strong dependence on the outer boundary condition; the radial power law for $\beta$ implies that we must specify its value at some fiducial radius; this in turn determines the value of $\beta$ in the interior (where the emissivity peaks). Secondly, the analytical solution assumes that, for $f=1$, constant $\beta$ corresponds to $\eta=0$ (no heating of particles). While correct for the self-similar solution of NY94, this is not strictly correct for global models of ADAFs (see below). This motivates us to consider global solutions of equations (26)-(28) and (30)-(31). The unsettling features of the analytical solution are not reproduced by these numerical calculations, although the general inference of subthermal magnetic fields is.

\subsection{Global Dynamics}

We have calculated global (transonic) ADAF models by solving equations (26)-(28) and (30)-(31) numerically using a relaxation method. Here we discuss the numerical method used; in $\S 4.2 .1$ we give sample solutions. The implications of these calculations are discussed in $\S 5$. The models discussed in this section are the generalization of NKH's global calculations to the case when particle heating is explicitly included.

We work in the pseudo-Newtonian potential introduced by Paczyński \& Wiita (1980), for which $\Omega_{K}^{2}=G M /\left[r\left(r-r_{g}\right)^{2}\right]$, where $r_{g}$ is the Schwarzschild radius and $M$ is the mass of the black hole. Integrating equation (26) implies $1=-\rho U_{r} r^{2} H_{\theta}$, where we normalize the density by taking the accretion rate to be $\dot{M}=4 \pi$. Using equation (26) and the expression for $H_{\theta}$ from above, we integrate equation (28) once to find

$$
\frac{d \Omega}{d R}=\frac{U_{r} \Omega_{K}\left(\Omega r^{2}-j\right)}{\alpha r^{2} v_{t}^{2}},
$$

where $j$ is the specific angular momentum accreted by the black hole.

Equations (27), (30)-(31), and (40) are the four first order differential equations which we solve (mass conservation simply normalizes the density). We also have two eigenvalues, $j$ and $r_{s}$, the location of the sonic point. We therefore require 6 boundary conditions.

Three of the boundary conditions are the values of $\Omega, c_{s}$, and $c_{t}$ (or equivalently, $\Omega, v_{t}$ and $\beta$ ) at the outer boundary (which we usually take to be at $r_{\text {out }}=10^{5} r_{g}$ ). We typically specify $\beta$ at the outer boundary and use the self-similar solution to calculate $\Omega, c_{s}$, and $c_{t}$.

Equations (26)-(28) and (30)-(31) can, using standard techniques, be manipulated to show the presence of a sonic point (at $\left.r=r_{s}\right)$, at which $d \ln \left|U_{r}\right| / d r=N / D$, where

$$
D=\frac{2 p+2}{p+2}-\frac{U_{r}^{2}}{v_{t}^{2}}
$$




$$
\begin{gathered}
N=\frac{r\left(\Omega_{K}^{2}-\Omega^{2}\right)}{v_{t}^{2}}-d\left(\frac{1}{r}-\frac{d \ln \Omega_{K}}{d r}\right)+\frac{\tilde{f} q^{+}}{\rho U_{r} v_{t}^{2}(p+2)}, \\
\tilde{f}=(\gamma-1)(\eta+f-1)+\left(\gamma_{t}-1\right)(\eta-1),
\end{gathered}
$$

and

$$
p=\frac{(\gamma-1) c_{s}^{2}+\left(\gamma_{t}-1\right) c_{t}^{2}}{v_{t}^{2}}
$$

A well-behaved solution must have $N=D=0$ at $r=r_{s}$, which provides two additional boundary conditions. The location of the sonic point is not predetermined but is our second eigenvalue (it is typically at $\sim 5 r_{g}$ for $\alpha \sim 0.3$ ).

The final boundary condition is the torque free condition, $j=\Omega r^{2}$, at the horizon. For numerical reasons, we typically apply this condition at $r=1.5 r_{g}$, but we have confirmed that the results are unchanged if we apply it at $r=1.1 r_{g}$. Applying this final boundary condition at the horizon is a defect of our acausal treatment of viscosity (see NKH for a discussion of this point and Gammie \& Popham 1998 for a causal treatment of viscosity in ADAF models).

\subsubsection{Results}

For the numerical results in this section we set $f=1, \gamma=5 / 3, \gamma_{t}=1.5$, and $\alpha=0.3$, and explore the solutions as a function of $\eta$ and the value of $\beta$ at the outer boundary. We focus on understanding the evolution of $\beta$ as a function of $r$, and the relationship between $\beta$ and $\eta$.

To start with, we apply the self-similar boundary condition corresponding to $\beta=1$ at $r=r_{\text {out }}$. Figure 1a shows the radial velocity $\left(U_{r}\right)$ and total sound speed $\left(v_{t}\right)$ for models ranging from $\eta=0$ to $\eta=1$. Figure $1 \mathrm{~b}$ shows the density for the same models. As predicted by the self-similar solution, the basic dynamical quantities (total pressure, density, etc.) are independent of the details of the particle heating (i.e., $\eta$ ). The insensitivity of $\rho, v_{t}$, and $U_{r}$ to $\eta$ is a generic feature of our models; we therefore do not show additional plots of these quantities.

Figures $1 \mathrm{c}$ and $1 \mathrm{~d}$ show $\Omega / \Omega_{K}$ and $\beta$ as functions of $r$ for $\eta$ varying from 0 to 1 in steps of 0.2. Here there is a nontrivial dependence on $\eta$. In order to understand the behavior of $\beta(r)$, the solid lines in Figure 2a show $\beta(r)$ for an alternative outer boundary condition, $\beta=15$ at $r=r_{\text {out }}$ (all other parameters are the same as in Figure 1).

The self-similar solution of $\S 4.1$ predicts that $\beta$ should be a monotonically increasing function of $r$, namely, $\beta \propto \beta_{\text {out }} r^{-2 a}$ where, for a fixed $\gamma_{t}, a \geq 0$ is just a function of $\eta$ (see eq. 38]). For $\eta \neq 1$, this is not always seen in the global calculations. In fact, in contrast to the self-similar solution, $\beta$ shows a tendency to approach a roughly constant value at small $r$ (especially near the sonic point, inside of which the fluid flows in nearly adiabatically), and to decrease with radius if it starts off with a large value (Fig. 2).

Deviations from self-similarity in the density and temperature profiles of the flow, driven

primarily by the existence of a sonic point, which modifies the radial velocity and therefore 
$\rho$, can lead to more noticeable changes in the entropy gradient (because the entropy gradient involves a cancellation between the temperature and density gradients). Since the value of $\eta$ is $\propto$ the entropy gradient (cf eqs. [30]-[32]), it, and hence $\beta$, is sensitive to such deviations. This is the basic reason why self-similarity is less accurate when calculating $\eta$ and $\beta$, even though it is reasonably good for $\rho, c_{s}^{2}$, etc..

Nonetheless, the key qualitative conclusion of the self-similar solution is confirmed by the global calculations. Explicit inclusion of particle heating at reasonable levels $(\eta \sim 1 / 2)$ leads to subthermal magnetic fields. In fact, there is an approximate mapping between the value of $\beta$ in the interior of the flow and $\eta$, which is independent of the outer boundary condition (this is in contrast to the self-similar solution, which was a power law in $r$, and thus very sensitive to the outer boundary condition). This is seen in Figure $2 \mathrm{~b}$, which shows $\beta(r)$ for $\eta=1 / 2$, taking $\beta_{\text {out }}=1,5,15$, and 50. In the inner portions of the flow, where the emissivity peaks, the solutions roughly converge to a common $\beta \sim 5$.

Furthermore, the global calculations confirm that strong levels of turbulence in the interior of the flow are incompatible with reasonable levels of particle heating. Strictly equipartition fields $(\beta=1)$ near $r \sim 1$ are only obtained for unphysically small values of $\eta \lesssim 0.1$ (Fig. 1d and $2 \mathrm{a})$.

\section{Discussion}

In $\S 2$ of this paper we derived the energy equations for particles, magnetic fields, and turbulence in optically thin, two temperature ADAFs (using mean field MHD). The fundamental energy equations are given in equations (5) - (12); for explicit calculations, however, we have used drastically simplified versions which approximate the turbulence as an additional fluid in the problem (the approximations required are detailed in $\$ 2.2$ ). One application of this analysis, given in Appendix A, is to resolve a disagreement in the literature between several treatments of the energetics of ADAFs.

The primary focus of this paper, however, has been to clarify and explore a previously underappreciated piece of physics regarding optically thin, two-temperature ADAFs. The issues addressed stem from the fact that non-relativistic ADAFs are, at some level, a singular problem. Strong advection leads to a virial flow in which non-relativistic particles tend to be isentropic. This is because, at least in the self-similar regime, being virial requires $T \propto \rho^{2 / 3}$, which is an adiabat for non-relativistic particles with $\gamma=5 / 3$. On the other hand, the turbulent heating of particles should cause the entropy of the gas to increase inwards. To resolve these conflicting requirements, either the flow must be non-rotating (unlikely) or else a formalism must be developed which allows for both differential rotation and particle heating.

This issue is particularly relevant for optically thin, two temperature, ADAFs which have been extensively applied to observed systems; for such flows, $T_{i} \gg T_{e}$ and the gas is quite accurately approximated as non-relativistic. Following NY95, a number of workers have suggested

that, because of the "singularity," a proper treatment of the flow dynamics should include the 
turbulent/magnetic contribution to the pressure and energy density (the electron contribution is expected to be less important if $\beta \lesssim T_{i} / T_{e}$, which is well satisfied in most models). The derivation in $\S 2$ "formalizes" this suggestion.

Previous attempts at phenomenologically including turbulence in the dynamics of ADAFs assumed a constant $\beta$ for the flow (e.g., NY95, EMN, Narayan et al. 1998a). What was not appreciated, however, is that this still does not account for the heating of particles; rather, the implicit assumption is that most of the energy generated by shear in the flow is stored as turbulent energy $(\S 3)$. This energy is in fact precisely what is required to maintain the constant $\beta$.

The scenario required by these models is unlikely to be realized in real flows. Strongly turbulent plasmas inevitably heat particles (energy cascades to small scales, etc.). To assess the implications of this, we impose particle heating in the dynamical equations by specifying that some fraction $(\equiv \eta)$ of the energy generated by turbulent stresses heats the particles. The remaining energy (a fraction $1-\eta$ ) is used to build up the magnetic field strength and turbulent kinetic energy as the gas flows in. The parameter $\eta$ therefore replaces the parameter $\beta$. The turbulence/magnetic field in the flow $(\beta)$ is now an output of the dynamical equations; it evolves so as to maintain the specified level of particle heating.

For thin accretion disks, one expects $\eta=1$ to good approximation due to the mismatch between the timescale on which the gas pressure increases (the inflow time) and the timescale on which shear acts to increase the turbulent energy (the rotational period). For $\alpha$ not $\ll 1$, no such mismatch exists for ADAFs and so $\eta \neq 1$, although we do expect $\eta \sim 1$ (rather than $\eta \ll 1 ; \S 2.3)$.

We have given analytical and numerical solutions for the dynamics of ADAFs at constant $\eta$. These are, respectively, the generalization of the NY94 self-similar solution and NKH and Chen et al.'s (1997) global calculations (in the Paczyński potential) to the case where particle heating is explicitly included. The results of the self-similar solution are particularly straightforward to understand. In previous, constant $\beta$, models, the constancy of $\beta$ was achieved at the expense of particle heating. If, instead, we require a reasonable level of particle heating, less energy is available for the turbulence and so $\beta$ must increase as the gas flows in. The turbulence/magnetic field therefore become subthermal. We emphasize, however, that the predicted density, radial velocity, and total pressure of the flow are relatively unchanged (with respect to NY94 or NKH) by the present analysis (§4; Figure 1).

Self-similarity requires that $\beta$ monotonically increase as the gas flows in, while our global calculations show that this is not reproduced if the turbulence is somewhat subthermal. The global calculations do confirm, however, that strong levels of turbulence $(\beta \sim 1)$ are incompatible with reasonable levels of particle heating (since they require $\eta \ll 1$; see Figures $1 \mathrm{~d}$ and 2a). If we prioritize particle heating, taking $\eta \sim 1 / 2$, we find that $\beta$ converges to $\sim 5$ in the interior of the flow, regardless of its initial value at large radii (Figure $2 \mathrm{~b}$ ).

Explicitly calculating $\beta$ requires understanding the non-linear saturation of the turbulence; this would provide the full profile, $\eta(r)$. Our point is that, regardless of the details of the nonlinear saturation, one expects that $\eta$ is not $\ll 1$. Taking a lower bound of $\eta \sim 1 / 2$ yields the 
corresponding bound, $\beta \gtrsim 5$.

The $\beta$ used in this paper refers to both the magnetic energy in the turbulence and the turbulent kinetic energy (cf eq. [14]); in particular, we assume roughly Alfvenic turbulence, for which these two energies are equal. Our analysis suggests that consistency with particle heating requires both subthermal magnetic and turbulent kinetic energies; on dimensional grounds, i.e., independent of the physics of angular momentum transport, this requires a relatively smaller $\alpha$. This can be seen by noting that $\alpha \approx-T_{r \phi} / \rho c_{s}^{2}$ in a Shakura \& Sunyaev-like closure relation, where $c_{s}$ is the sound speed. From the definition of the stress tensor (eq. [12]), we expect $T_{r \phi} \lesssim \rho c_{t}^{2}$, where $c_{t}^{2}=c_{s}^{2} / \beta$ is the square of the turbulent velocity. Dimensional analysis therefore implies $\alpha \lesssim 1 / \beta$. Quantitatively, then, the constraint $\beta \gtrsim 5$ suggests that $\alpha \lesssim 0.2$.

\subsection{Caveats}

To conclude, we discuss several key assumptions implicit (or explicit) in our analysis.

First, the correct value for $\eta(r)$ is highly uncertain, and the inferred $\beta$ depends nontrivially on the $\eta$ chosen (see Figures $1 \& 2$ ). For example, one might think that $\eta$ and $\beta$ would be anti-correlated (i.e., preferential build up of the turbulence if it is subthermal). In principle, one could solve the mean field energy equations (eqs. [5] - [7]) using, e.g., a second order closure scheme, and thus calculate $\eta$ (Speziale 1991), but this is well beyond the scope of our paper.

Second, we treat the turbulence/magnetic fields in the flow using a crude fluid model. Even within the context of this model, we assume that the effect of flux freezing/compression on the turbulent energy is insufficient to bring the turbulence into equipartition with the gas $\left(\gamma_{t} \lesssim 5 / 3\right)$. This requires that less than half of the magnetic energy is in radial perturbations and that more than a third of the turbulent kinetic energy is in radial perturbations (Appendix B). Our physical picture is that the primary source of the turbulent energy is shear in the mean flow.

A noticeable omission is our neglect of third order (and higher) turbulent quantities (§2.2). These represent, among other things, the turbulent diffusion of energy/entropy, and thus are quite relevant for the issues discussed in this paper. Unfortunately, turbulent transport is particularly complicated in ADAFs, since both convection and MHD turbulence are believed to be important.

Although we consider a radially varying $\beta$, we take $\alpha$ to be a constant. Aside from simplicity, our motivation for this restriction is that there is generally no self-similar advectiondominated accretion solution when $\alpha$ decreases as the gas flows in (which would be expected by our analysis if $\alpha \propto \beta^{-1}$ ). This is because a decreasing $\alpha$ leads to an increase in density more rapid than $r^{-3 / 2}$ (if $\alpha \propto r^{g}, U_{r} \propto r^{-1 / 2+g}$, and $\rho \propto r^{-3 / 2-g}$ ). The compressive heating associated with this density profile is sufficiently large that it generally yields a decreasing entropy gradient for the flow. The only viable self-similar solution in this case is one which is cooling dominated, rather than advection-dominated. 
Finally, our calculations are (as is standard) a one dimensional (height integrated) analysis of a three dimensional problem. Relaxing this restriction will likely make the singularity inherent in the dynamical equations less severe. The extent to which this will alleviate our concerns about particle heating is, of course, unclear.

Acknowledgments. It is a pleasure to thank Charles Gammie and Andrei Gruzinov for useful discussions. EQ was supported by an NSF Graduate Research Fellowship; this work was also supported by NSF Grant AST 9423209. 


\section{A. Related Work}

Here we compare our analysis of the energetics of ADAFs with several other treatments in the literature, and clarify several issues about the use of "effective" adiabatic indices.

EMN treat the energetics of an ADAF using both a total energy equation and an electron energy equation. Our derivation/discussion of the total energy equation in $\S 2$ is a more rigorous treatment of the problem, which arrives at conclusions similar to theirs (which is based on Esin 1997). The one difference is that they neglect the turbulent velocity (i.e., $\beta_{v} \rightarrow \infty$ ), so that their expressions for $c_{t}^{2}$ and $\gamma_{g}$ are slightly different (the most general expression for $\gamma_{g}$ is given by eq. [B6] of Appendix B).

EMN's treatment of the electron energy equation (their Appendix A) is, however, quite different from ours. They take the electron internal energy and pressure to be effective quantities, containing contributions from the magnetic field. They thus argue that the adiabatic index which shows up in the electron energy equation is also an effective $\gamma$ (in fact they take $\gamma_{e}=\gamma_{g}$ ). The physical picture on which this is based is that (1) all of the energy generated by turbulent stresses heats the particles, i.e., $H=q^{+}$, and (2) the energy required to bring the magnetic energy from its flux-frozen value to the equipartition value comes from the thermal energy of the particles (hence $\gamma_{e}$ smaller than its monatomic value). The problem with this analysis is that it incorrectly treats the thermal energy of the particles as a source of magnetic energy. Within the context of MHD, turbulence and magnetic fields can act as a source of thermal energy, while the converse is not possible (this can be seen formally using the mean field energy equations in $\S 2$ ). If we set $H_{e}=0$ (no turbulent heating of electrons), then the turbulence and magnetic field should not appear in the electron energy equation (except as a third order turbulent diffusion term), while in EMN's treatment they still would (through the effective $\gamma$ ). To conclude, then, the appropriate adiabatic index in the electron energy equation is not an "effective" one, but rather that of a monatomic ideal gas.

Manmoto et al. (1997) and Nakamura et al. (1997) also solve the energy equations for ADAFs. They take electron and ion equations analogous to (21) and (22) as their basic energy equations, with $H_{e}=0, H_{i}=q^{+}$, and $\gamma_{e}=\gamma_{i}=5 / 3$. Setting $H_{e}=0$ assumes no turbulent heating of electrons, which we regard as overly optimistic. Setting $\gamma_{e}=5 / 3$ is correct in spirit (in that there is no "turbulent" component to $\gamma_{e}$ ), but it assumes non-relativistic electrons; this is incorrect in the inner $\sim 10^{2}-10^{3}$ Schwarzschild radii. Setting $H_{i}=q^{+}$assumes that all of the energy generated by turbulent stresses heats the ions. As emphasized in this paper, however, $H$ is, in general, $\neq q^{+}$(although it is likely $\sim q^{+}$).

There is a close relationship between the "ion" and "total" energy equations discussed above and in $\S 2$. In particular, in treating the "ion/total" energy equation, one can either use a monoatomic adiabatic index or an effective adiabatic index. If the former, the heating term should be $H \neq q^{+}$, since one is truly writing a particle energy equation. If the latter, the heating term should be $q^{+}$(see eq. [15]). Put another way, it is precisely the inequality of $H$ and $q^{+}$which allows one to use an effective adiabatic index. This can be seen explicitly by "solving" the turbulent energy equation (eq. [24]) for $H$ and substituting it into the particle energy equation (eq. [7]), in which case one readily derives the total energy equation (eq. [15]). 
No such analysis is allowed for the electron energy equation, however, since it is a standard assumption of ADAF models that $T_{e} \ll T_{i}$ and the electrons couple poorly to the turbulence $\left(\delta_{H} \ll 1\right)$. The uncertainty introduced by $H \neq q^{+}$is therefore absorbed into an uncertainty in (the already uncertain) electron heating parameter, $\delta_{H}$, rather than in the adiabatic index.

The above arguments regarding the electron adiabatic index in ADAFs break down if the distribution functions are anisotropic. In this case adiabaticity cannot be described by a single adiabatic index (cf double adiabatic theory). In particular, for anisotropic distribution functions, thermal energy can be a source of magnetic energy (the fire-hose instability being a well known example), contrary to the arguments using MHD given above. ADAFs are nearly collisionless systems; isotropy is therefore not guaranteed. Nonetheless, we (as with all other workers in the field) have proceeded on the assumption that it is a reasonable approximation. Anisotropic distribution functions are known to be unstable to generating modes (e.g., Alfven waves, fast modes, whistlers) which act to restore isotropy (e.g., Melrose 1980). The timescale for such processes is typically of order the cyclotron period, much shorter than the dynamical time in the accretion flow.

\section{B. Anisotropic Turbulence}

When simplifying the mean field energy equations in $\S 2$, we assumed that the diagonal components of the turbulent stress tensor are all equal (i.e., $b_{r}^{2} \approx b_{\phi}^{2} \approx b_{\theta}^{2}$, etc.). This is equivalent to assuming that the "turbulent pressure" is isotropic. In this Appendix, we generalize the derivation of $\S 2$ to arbitrary diagonal components for the stress tensor.

Assuming azimuthal symmetry, $U_{\theta}=0$, and uniform rotation on spherical shells, but allowing for arbitrary values of $T_{r r}, T_{\theta \theta}$, and $T_{\phi \phi}$, the energy generation term of equation (11), which shows up in the total energy equation for the fluid, becomes

$$
G=-\left(\left\langle\frac{1}{2} \rho u^{2}\right\rangle+2\left\langle\frac{b^{2}}{8 \pi}\right\rangle+\gamma\langle\epsilon\rangle\right) \partial_{k} U_{k}-T_{r r} \frac{d U_{r}}{d r}-\left(T_{\theta \theta}+T_{\phi \phi}\right) \frac{U_{r}}{r}-G_{o d},
$$

where

$$
G_{o d}=T_{r \phi} \sin \theta r \frac{d \Omega}{d r}-T_{\theta \phi} \Omega \cos \theta
$$

is the contribution to $G$ from the off-diagonal components of the stress tensor. This is the same as derived in $\S 2.2$.

For $T_{r r}=T_{\theta \theta}=T_{\phi \phi}$, as taken in $\S 2$, equation (B1) reduces to equation (18). This is because $d U_{r} / d r+2 U_{r} / r=\nabla \cdot \mathbf{U}$ under the assumptions of $U_{\theta}=0$ and azimuthal symmetry. In this case, the terms associated with geometrical convergence of the flow in spherical coordinates (the $d U_{r} / d r$ and $U_{r} / r$ terms) are equivalent to a term $\propto d \rho / d r$, i.e., a $P d V$ work term.

As equation (B1) shows, however, one cannot in general write the "geometrical" terms as "compressive" terms and thus one cannot derive a fluid approximation for the turbulence in the flow. Assuming self-similarity, $U_{r} \propto r^{-1 / 2}$, allows one to do so. In this case, $d U_{r} / d r \approx-\nabla \cdot \mathbf{U} / 3$ and $U_{r} / r \approx 2 \nabla \cdot \mathbf{U} / 3$. With this simplification, equation (B1) reduces to 


$$
G=-\left[\left(\frac{7}{3}-2 h_{v}\right)\left\langle\frac{1}{2} \rho u^{2}\right\rangle+\left(\frac{2}{3}+2 h_{b}\right)\left\langle\frac{b^{2}}{8 \pi}\right\rangle+\gamma\langle\epsilon\rangle\right] \partial_{k} U_{k}-G_{o d}
$$

where

$$
h_{v}=\frac{\left\langle v_{r}^{2}\right\rangle}{\left\langle v^{2}\right\rangle} \text { and } h_{b}=\frac{\left\langle b_{r}^{2}\right\rangle}{\left\langle b^{2}\right\rangle}
$$

are the fraction of the turbulent kinetic energy $\left(h_{v}\right)$ and magnetic energy $\left(h_{b}\right)$ in radial perturbations, respectively.

Expressing the level of turbulence in the flow in terms of the $\beta$ 's of equation (14) allows one to derive a total energy equation for the flow which is identical in form to equation (15). The expressions for the total sound speed, $v_{t}$, and the effective adiabatic index, $\gamma_{g}$, are, however, different. They are now given by

$$
v_{t}^{2}=c_{s}^{2}\left[1+\beta_{v}^{-1}\left(\frac{4}{3}-2 h_{v}\right)+\beta_{b}^{-1}\left(2 h_{b}-\frac{1}{3}\right)\right]
$$

and

$$
\gamma_{g}=\frac{\gamma+(\gamma-1) \beta_{v}^{-1}\left(\frac{7}{3}-2 h_{v}\right)+(\gamma-1) \beta_{b}^{-1}\left(\frac{2}{3}+2 h_{b}\right)}{1+(\gamma-1) \beta_{v}^{-1}+(\gamma-1) \beta_{b}^{-1}} .
$$

If we set $h_{v}=h_{b}=1 / 3$ and $\beta_{b}=\beta_{v} \equiv \beta$, equations (B5) and (B6) reduce to equations (16) and (17), respectively.

Equation (B6) demonstrates the simple, but important, point that the effect of flux freezing/compression on the turbulent energy depends on the geometry of the turbulence. If we set $\beta_{b} \rightarrow 0$ in equation (B6), we recover the "adiabatic index" associated with flux freezing of the magnetic field, namely

$$
\gamma_{b}=\frac{2}{3}+2 h_{b}
$$

The corresponding adiabatic index for the velocity component of the turbulence is

$$
\gamma_{v}=\frac{7}{3}-2 h_{v}
$$

It should be emphasized again that equations $(\overline{\mathrm{B} 3})-(\overline{\mathrm{B} 8})$ are strictly valid only in the self-similar regime.

In the bulk of this paper we have assumed that flux freezing is insufficient to keep the magnetic field/turbulence in equipartition with the gas, i.e., that $\gamma_{b} \lesssim 5 / 3$. By equation (B7), this can be recast as an assumption on the magnetic field geometry, namely $h_{b} \lesssim 1 / 2$, i.e., less than half of the magnetic energy is in radial perturbations. The corresponding constraint on the turbulent velocity field is that $h_{v} \gtrsim 1 / 3$, i.e., more than $1 / 3$ of the turbulent kinetic energy is in radial perturbations.

Both of these constraints $\left(h_{b} \lesssim 1 / 2\right.$ and $\left.h_{v} \gtrsim 1 / 3\right)$ are satisfied in numerical simulations of thin accretion disks (cf Hawley, Gammie, \& Balbus 1996). These are, however, of questionable relevance for the accretion flows at hand. For laminar, non-rotating, spherical accretion, it 
is known that $h_{b} \sim 1$ : the magnetic field becomes purely radial (Shapiro 1973), and flux freezing is energetically important, invalidating the analysis of this paper. This result is also of questionable relevance for the accretion flows at hand since, while quasi-spherical, ADAFs are differentially rotating, turbulent, etc.. We suspect that ADAFs lie somewhere between the two extremes of thin disks and spherical accretion, but numerical simulations will be necessary to determine precisely where. 


\section{References}

Abramowicz, M., Chen, X., Granath, M., \& Lasota, J.-P., 1996, ApJ, 479, 179

Abramowicz, M., Chen, X., Kato, S., Lasota, J.-P., \& Regev, O., 1995, ApJ, 438, L37

Abramowicz, M., Lanza, A. \& Percival, M. J., 1997, ApJ, 479, 179

Balbus, S. A., \& Hawley, J.F., 1998, Rev. Mod. Phys., 70, 1

Bisnovatyi-Kogan, G. S., \& Lovelace R. V. E., 1997, ApJ, 486, L43

Chen, X., Abramowicz, M.A., Lasota, J.-P., Narayan, R., \& Yi, I. 1995, ApJ, 443, L61

Chen, X., Abramowicz, M.A., Lasota, J.-P., 1997, ApJ, 476, 61

Esin, A. A., 1997, ApJ, 482, 400

Esin, A. A., McClintock, J. E., \& Narayan, R., 1997, ApJ, 489, 86 (EMN)

Gammie, C.F \& Popham, R.G., 1998, ApJ, 498, 313

Hawley, J. F., Gammie, C. F., \& Balbus, S. A., 1996, ApJ, 464, 690

Ichimaru, S. 1977, ApJ, 214, 840

Kato, S., Abramowicz, M., \& Chen, X. 1996, PASJ, 48, 67

Kato, S., Fukue, J., Mineshige, S., 1998, Black-Hole Accretion Disks (Japan: Kyoto University Press)

Manmoto, T., Mineshige, S., \& Kusunose, M., 1997, ApJ, 489, 791

Melrose, D.B. 1980, Plasma Astrophysics (New York: Gordon \& Breach)

Nakamura, K. E., Masaaki, K., Matsumoto, R., \& Kato, S. 1997, PASJ, 49, 503

Narayan, R., Barret, D., \& McClintock, J. E., 1997, ApJ, 482, 448

Narayan, R., Kato, S., \& Honma, F. 1997, ApJ, 476, 49 (NKH)

Narayan, R., \& Yi, I., 1994, ApJ, 428, L13 (NY94)

Narayan, R., \& Yi, I., 1995a, ApJ, 444, 231

Narayan, R., \& Yi, I., 1995b, ApJ, 452, 710 (NY95)

Narayan, R., Mahadevan, R., Grindlay, J.E., Popham, R.G., \& Gammie, C., 1998a, ApJ, 492, 554

Narayan, R., Mahadevan, R., \& Quataert, E., 1998b, in The Theory of Black Hole Accretion Discs, eds. M.A.

Abramowicz, G. Bjornsson, and J.E. Pringle (Cambridge: Cambridge University Press)

(astro-ph/9803131)

Paczyński, B. \& Wiita, P. J., 1980, A\&A, 88, 23

Parker E.N. 1979, Cosmical Magnetic Fields (New York: Oxford University Press)

Rees, M. J., Begelman, M. C., Blandford, R. D., \& Phinney, E. S., 1982, Nature, 295, 17

Shakura, N. I., \& Sunyaev, R. A., 1973, A\&A, 24, 337

Shapiro, S. L., 1973, ApJ, 185, 69

Shapiro, S. L., Lightman, A. P., \& Eardley, D. M. 1976, ApJ, 204, 187

Speziale, C. G., 1991, Ann. Rev. Fluid Mech., 23, 107 

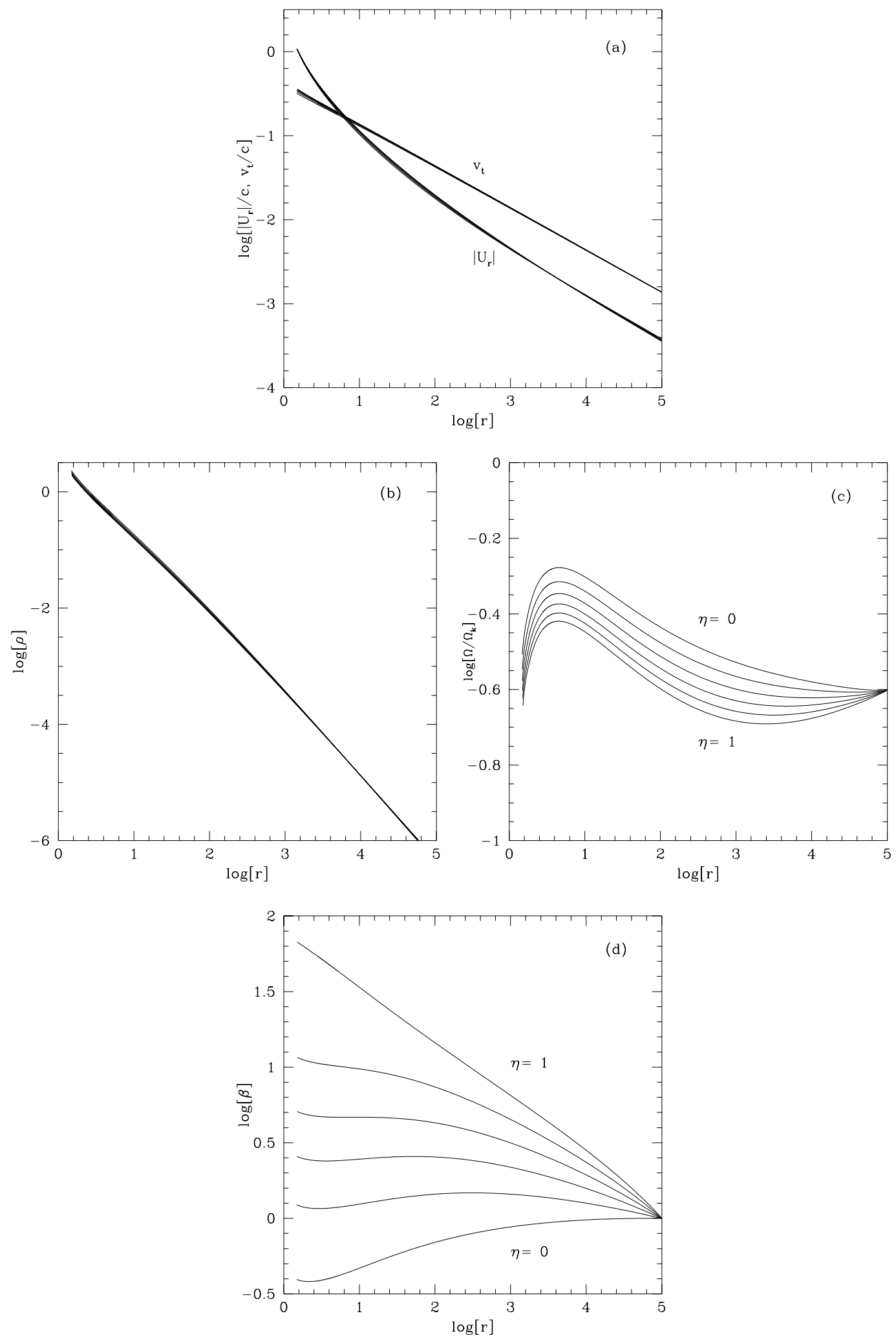

Fig. 1.- Radial profiles of several quantities in our global dynamical models of ADAFs. Each panel shows plots for $\eta$ from 0 to 1 in steps of 0.2 . Other parameters take the values: $\gamma_{t}=1.5, \alpha=0.3, f=1$, and $\beta=1$ at $r=r_{\text {out }}=10^{5} r_{g}$. (a) radial velocity $\left(U_{r}\right)$ and total sound speed $\left(v_{t}\right),(\mathrm{b})$ density, (c) rotation rate in units of the Keplerian rate, (d) ratio of gas to turbulent pressure $(\beta)$. Note that $U_{r}, v_{t}$, and $\rho$ are essentially independent of $\eta$, while $\Omega / \Omega_{K}$ and, in particular, $\beta$, depend non-trivially on $\eta$. 

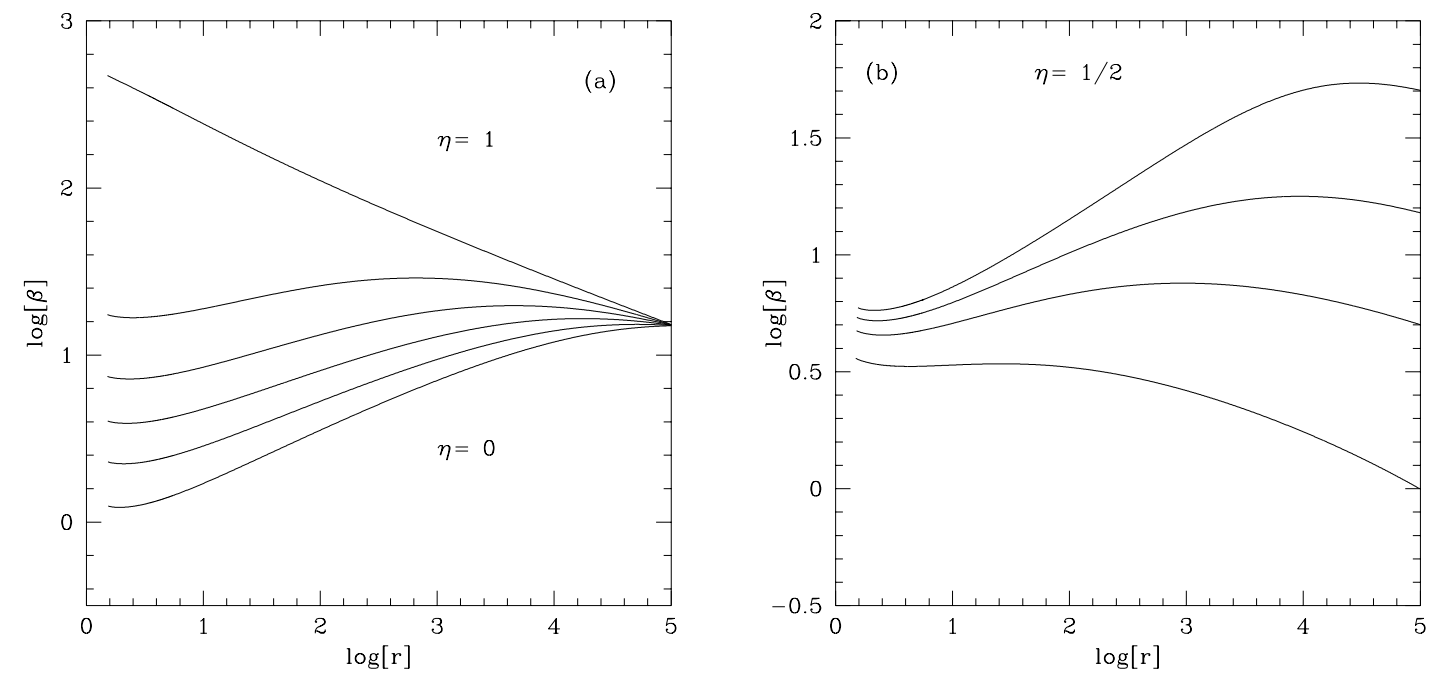

Fig. 2.- (a) $\beta(r)$ for the same parameters as in Figure 1, except $\beta$ is set to 15 at the outer boundary. (b) $\beta(r)$ for $\eta=1 / 2, \gamma_{t}=1.5$, and several different values of $\beta$ at the outer boundary. The solutions roughly converge to a common $\beta \sim 5$ in the interior (although the radial variation of $\beta$ is quite different). 